\title{
Wie lässt sich der Alltag mit Psoriasis bewältigen?
}

- Bei einer Psoriasis leidet nicht nur die Haut, sondern häufig auch die Seele. Kein Wunder, dass Patienten viele Fragen haben. Eine neue kostenlose Patientenbroschüre hilft dabei, diese kompetent zu beantworten.

Um die Krankheit zu akzeptieren und auch in schwierigeren Zeiten die Behandlung konsequent fortzuführen, ist eine gute Aufklärung wichtig. Denn je besser der Patient informiert ist, desto eher kann er in die Therapie eingebunden werden und dabei helfen, die Behandlung erfolgreich umzusetzen.

Die Patientenbroschüre „20 Fragen - 20 Antworten“ zum Thema „Psoriasis - Schuppenflechte" beantwortet auf knapp 30 Seiten die häufigsten Fragen, die im Zusammenhang mit der Schuppenflechte, ihrer Therapie und der psychosozialen Auswirkungen immer wieder von Betroffenen gestellt werden:
Woher kommt die Erkrankung? Wie kann ich sie durch die richtige Lebensführung günstig beeinflussen? Welche Therapiemöglichkeiten gibt es? Welche zusätzlichen Informationsmöglichkeiten gibt es? Da die Psoriasis sich oft massiv auf den Alltag auswirkt, möchten Patienten gerade hierzu viele Dinge wissen.

Für eine praxis- und lebensnahe Ausrichtung hat der Deutsche Psoriasisbund das Projekt unterstützt. Ermöglicht wurde es durch die Firma LEO Pharma, die mit der Veröffentlichung dieser Broschüre - gemäß ihrem Firmenleitbild - die Lebensqualität von Menschen mit Psoriasis verbessern möchte.

Durch die Broschüre sollen die wichtigsten Probleme schon im Vorfeld dargestellt und erläutert werden, sodass in der Sprechstunde mehr Zeit für gezieltere Fragen bleibt. Die kostenlose Broschüre kann von allen Ärzten (immer im Paket mit jeweils 50 Exemplaren) fürs Wartezimmer bestellt werden. Bestellungen an: vertrieb@springer. com oder per Fax an 06102/506240, Stichwort "20 Fragen - Psoriasis".

Volker Schuck .

\section{Demenz}

\section{Prävention und Therapie}

— Vor dem Hintergrund des demografischen Wandels ist die Prävention und Therapie der Alzheimerdemenz heute mehr denn je relevant, betonte Prof. Hans Förstl, München. Wichtige vermeidbare Risikofaktoren sind bereits bekannt, wie Rauchen, Alkoholkonsum, schlecht eingestellter Diabetes, körperliche Inaktivität, Hypertonus, Depression, ungesunde Lebensweise oder Übergewicht. Ist die Alzheimerdemenz eingetreten, sollte sie unverzüglich behandelt werden.

\section{Symptomatische Besserung möglich}

Die aktuelle Leitlinie Demenz der World Federation der Fachgesellschaften für Biologische Psychiatrie (WFSBP) diskutierte dazu die verschiedenen Therapieoptionen: „Wir haben hinreichende Daten, die eine symptomatische Besserung belegen. Deswegen erfüllen sie auch Evidenzlevel B, Empfehlungsgrad 3", fasste Prof. Ralf Ihl, Düsseldorf, zusammen. Die einzelnen zur Verfügung stehenden Antidementiva wie Azetylcholinesterasehemmer, Ginkgo biloba oder Memantine haben einen moderaten Effekt für eine begrenzte Zeit bei einem Teil der behandelten Menschen. „Das klingt nach wenig, aber für den Einzelnen kann es viel bringen".

So zeigten gerade die 20-Jahres-Daten mit Ginkgo biloba, dass diejenigen, die das Ginkgo-Präparat EGb761 ${ }^{\circledR}$ (Tebonin ${ }^{\oplus}$ ) einnahmen, eine signifikant geringere Abnahme der mentalen Leistung aufwiesen als unbehandelte Personen oder Menschen, die Piracetam eingenommen hatten [Amieva $\mathrm{H}$ et al. PLoS ONE 2013; 8: e52755]. Daneben stieg auch die Überlebensrate (signifikant im Vergleich zu Placebo).

\section{Dr. Katrin Wolf .}

\footnotetext{
- Quelle:DGGPP-Kongress - Mittagssymposium "Demenzen III: Kontroversen in der Prävention und Therapie". Essen, Juni 2013 (Veranstalter: Dr. Wilmar Schwabe)
}

\section{Kurz notiert}

Unkomplizierte Harnwegsinfekte - Wie die Deutsche Gesellschaft für Allgemein und Familienmedizin (DEGAM) in der S3-Leitlinie festhält, muss bei akuter unkomplizierter Zystitis nicht immer eine Antibiotikatherapie begonnen werden. Wegen der zunehmenden Resistenz der Erreger ist auch eine rein symptomatische Therapie oder der Einsatz alternativer Methoden eine vertretbare Therapieoption.

Interessant ist in diesem Zusammenhang eine In-vitro-Untersuchung, in der an isolierten Blasenstreifen durch die Zugabe eines Phytopharmakons $\left(\right.$ Canephron ${ }^{\circledR} \mathrm{N}$ ) eine relaxierende Wirkung gemessen werden konnte. Die Spasmolyse beruht hierbei hauptsächlich auf den Heilpflanzen Liebstöckel und Rosmarin. Das Präparat enthält darüber hinaus auch Tausendgüldenkraut.

Red. -

- Nach Informationen des Unternehmens Bionorica SE 